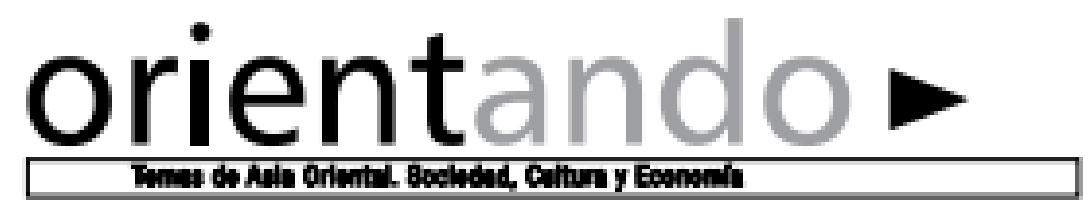

Año 10. Número 20. Abril 2020-Septiembre 2020

ISSN 2007-5723

Centro de Estudios China-Veracruz/Universidad Veracruzana

\title{
Informe anual de actividades septiembre2019-agosto 2020
}

Annual activity report september 2019-august 2020

Aníbal Carlos Zottele

\section{Introducción}

El Centro de Estudios China-Veracruz (Cechiver) de la Universidad Veracruzana (UV) ubicado en la Unidad de Servicios Bibliotecarios y de Información (USBI) de la ciudad de Xalapa, Ver., ha sido distinguido con la ampliación de su dependencia con una nueva oficina ubicada en la Facultad de Contaduría en el Campus Veracruz-Boca del Río, inaugurada en 2019 por el doctor Alfonso Gerardo Pérez Morales, vicerrector de la región.

Nuestra entidad, creada por resolución del Consejo Universitario General el 23 de septiembre de 2008, se ha dedicado a las actividades de investigación, a la transmisión social de los productos de ese cometido, al fortalecimiento del proceso de enseñanza-aprendizaje, y al desarrollo de las tareas de internacionalización de la UV articulando vínculos y cooperación científica y docente, con instituciones de la región Asia-Pacífico y especialmente con China.

Cechiver ofrece tres programas educativos: Maestría en Economía y Sociedad de China y América Latina, el Diplomado Virtual sobre Cultura y Negocios en China y el Diplomado Negocios entre China y México que se ha dictado en trece oportunidades en diferentes sedes del país asiático, destacando las ciudades de Beijing, Nanjing, Shanghái, Changzhou, Chongqing, Tianjin y Baoting. 
Nuestro Centro edita la Revista Orientando Temas de Asia Oriental, Sociedad, Cultura y Economía, que este 2020 cumple diez años de su primera publicación de frecuencia semestral.

A continuación se presenta el informe de actividades septiembre 2019-agosto 2020 en atención a la Misión, Visión, Valores, Objetivos y Funciones que el Honorable Consejo Universitario estableció con motivo de la creación de Cechiver y a los tres ejes, sus programas estratégicos y líneas de acción, que guían el Programa de Trabajo Estratégico 2017-2021. Pertenencia y pertinencia.

\section{Misión de Cechiver}

Ser un espacio permanente de investigación, análisis, discusión, promoción y difusión de propuestas de intercambios científico, académico, cultural y económico de México, en especial del estado de Veracruz, con la República Popular de China.

\section{Visión}

Contribuir al fomento de las relaciones socioeconómicas entre las instituciones y organismos de México y China mediante actividades de investigación, docencia, difusión y vinculación.

\section{Valores}

Tolerancia: Se reconocen las actitudes y características de la comunidad universitaria, respetando las diferencias y la diversidad en busca de la igualdad y no discriminación.

Respeto: Se aprecia la dignidad de las personas y se tratan con equidad y cordialidad.

Responsabilidad: Se realizan las funciones que la sociedad requiere para su desarrollo y se responde por los actos propios.

Compromiso: Se cumple oportunamente con nuestros objetivos con total dedicación y dignidad.

Solidaridad: Se une nuestro esfuerzo a causas que contribuyen al bienestar de la sociedad.

Orientando. Temas de Asia Oriental. Sociedad, Cultura y Economía. Revista editada por el Centro de Estudios China-Veracruz de la Universidad Veracruzana, México (Centro de Estudios APEC) / año 10 / número 20/ 
Empatía: Se asume una actitud receptiva sobre la realidad que afecta a otros y la transformamos en una oportunidad de cambio.

Lealtad: Se promueve el sentido de pertenencia a nuestra institución con acciones que favorezcan su desarrollo.

Trabajo en equipo: Se establece el trabajo colaborativo para alcanzar el éxito colectivo por encima del reconocimiento individual.

Honestidad: Se incentiva el ejercicio de una actitud a favor de la verdad en relación con el entorno a través de un comportamiento recto y honrado.

Igualdad y no discriminación: Se reconocen los méritos y derechos de cada integrante de la comunidad universitaria sin distinción de género, aceptamos la riqueza que ofrece la diversidad y actuamos con rectitud e imparcialidad para dar un trato justo sin importar condiciones o circunstancias en particular.

Equidad: Se reconoce como iguales a cada integrante de la comunidad universitaria sin otorgar un trato preferencial o perjudicial para cualesquiera de ellos.

Transparencia: Se reconoce el derecho de las personas de tener acceso a la información clasificada como pública, facilitando los medios disponibles para que todo solicitante pueda acceder a ella de manera confiable y oportuna.

Objetividad: Se actúa con base en datos y hechos comprobables, manteniendo aparte opiniones, simpatías y preferencias personales.

Integridad: Se actúa con discreción en el manejo de información y congruencia entre el pensamiento, sentimiento y acción, sin afectar los derechos de otras personas.

\section{Objetivos generales}

- Crear los espacios multidisciplinarios para la investigación sobre China y su impacto en nuestra sociedad.

Orientando. Temas de Asia Oriental. Sociedad, Cultura y Economía. Revista editada por el Centro de Estudios China-Veracruz de la Universidad Veracruzana, México (Centro de Estudios APEC) / año 10 / número 20/ 
- Impulsar la confianza, la comprensión y la cooperación científica, cultural y económica entre Veracruz y China.

- Promover la distribución social del conocimiento sobre las experiencias económicas y sociales de China.

\section{Funciones}

Investigaciones multidisciplinarias referidas a la cultura china, incluyendo el estudio de su idioma, literatura, historia, relaciones internacionales y demás aspectos sociales y económicos.

Investigaciones del impacto de las políticas y la actividad económica de China sobre México, y otras áreas de América Latina y del Caribe. Influencia de nuestro subcontinente sobre la economía y sociedad de China. Evaluación de las relaciones bilaterales y regionales con este país.

Promoción y realización de intercambios académicos con instituciones de educación superior de México y universidades e instituciones de China.

Realización de cursos, conferencias, coloquios y publicaciones relacionadas con los temas de investigación.

Evaluación de oportunidades para los pequeños y medianos empresarios, en materia de inversiones, comercio y cooperación con sus pares chinos.

Colaboración con distintas instancias académicas de la UV para la enseñanza de la cultura, ciencia, tecnología, economía y sociedad de la República Popular China.

Desde 2010 se incorporaron a las funciones de Cechiver, las investigaciones sobre otras regiones de Asia-Pacífico y se desarrollaron vínculos con instituciones académicas de esa región.

Desde entonces se realizaron gestiones que culminaron con buenos resultados para que nuestro Centro sea reconocido por las Secretarías de Economía y de Relaciones Exteriores Orientando. Temas de Asia Oriental. Sociedad, Cultura y Economía. Revista editada por el Centro de Estudios 
de México como Centro de Estudios de APEC y luego de su aprobación se obtuvo el reconocimiento internacional.

\section{Ejes y líneas de acción. Programa de Trabajo Estratégico 2017-2021}

\section{Eje 1: Liderazgo académico}

A través de este eje estratégico se busca fortalecer y articular las funciones de docencia e investigación, promoviendo la innovación y buscando la excelencia para la formación integral y armónica en lo profesional, intelectual, social y humana del estudiante, como eje y razón de ser de la Institución.

Este eje es rector de toda nuestra actividad académica, puesto que, salvo las experiencias educativas que corresponden a áreas básicas, son resultado de investigaciones desarrolladas en forma individual o en cooperación con instituciones académicas nacionales y/o del exterior.

a) Oferta educativa de calidad

El objetivo de las múltiples labores que realiza Cechiver en correspondencia a este eje, es diversificar y actualizar la oferta educativa con calidad, equidad, eficacia, eficiencia y pertinencia para el desarrollo regional y nacional, considerando las diversas modalidades de enseñanza y haciendo uso de las tecnologías de información y comunicación en el aprendizaje.

Desde su inicio, la principal característica que se propuso y desarrolló el Cechiver, fue el dictado de experiencias educativas en forma virtual. Para lo cual el Centro se valió del conocimiento y aplicación sistemáticos de la plataforma EMINUS.

En el periodo de este informe, la Maestría en Economía y Sociedad de China y América Latina (MESCAL), participó en la convocatoria de ingreso al Posgrado UV-II comprendida

Orientando. Temas de Asia Oriental. Sociedad, Cultura y Economía. Revista editada por el Centro de Estudios China-Veracruz de la Universidad Veracruzana, México (Centro de Estudios APEC) / año 10 / número 20/ 
del 27 de agosto al 27 de septiembre de 2019, en la cual se alcanzó un total de 11 aspirantes, lo que significó un aumento del 22\% en comparación con el ejercicio anterior.

Después de llevar a cabo el proceso de selección, donde se evaluó a los aspirantes; fueron aceptados 8 de ellos ( $72 \%$ del total de solicitantes)

De esta manera, durante el período evaluado, el Posgrado está desarrollando experiencias educativas para dos generaciones.

Como se ha indicado todas las experiencias educativas se han impartido de forma virtual y sin contratiempos, a través de la plataforma EMINUS, contando a la fecha con 4 generaciones que han recibido su educación y formación on-line. Además, se han incorporado algunas otras herramientas, como zoom, que han servido para nutrir el trabajo entre estudiantes y maestros.

Cada una de las experiencias educativas es implementada en consideración del perfil deseado de nuestros futuros maestros en Economía y Sociedad de China y América Latina, por lo que los contenidos son actuales y relevantes para las necesidades y competencias que impone el proceso de globalización.

Por otro lado, se continúa trabajando en la internacionalización del Posgrado, que hasta el momento ha contado con algunos aspirantes que radican en el extranjero, uno de los cuales participó en la convocatoria del periodo 2019-II.

Así mismo, la presencia y reconocimiento de la Maestría es cada vez mayor entre la comunidad universitaria, por lo que la movilidad de otros Posgrados hacia MESCAL se ha incrementado en un 50\% en comparación con el semestre inmediato anterior (agosto 2019enero 2020).

\section{b) Planta Académica}

El objetivo en esta línea de acción es contar con una planta académica con altos estándares profesionales y con formación pedagógica de acuerdo con el Modelo Educativo Institucional,

Orientando. Temas de Asia Oriental. Sociedad, Cultura y Economía. Revista editada por el Centro de Estudios China-Veracruz de la Universidad Veracruzana, México (Centro de Estudios APEC) / año 10 / número 20/ 
que garantice la enseñanza en contextos reales, sea líder en producción académica, redes de colaboración, grupos colegiados y generación y aplicación del conocimiento. Que cuente con un perfil apropiado para el cumplimiento de sus funciones, en el marco de los valores y principios institucionales; organizados en estructuras colegiadas de docencia e investigación que den sustento a la planeación y evaluación académica, el desarrollo de los programas educativos y las líneas de investigación.

Las actividades están encaminadas a fortalecer nuestra plantilla docente, actualmente conformada por 14 profesores. En el periodo comprendido de septiembre 2019 a agosto 2020, se logró incorporar a 2 maestros, hasta el momento el $23.07 \%$ de sexo femenino (3) y el $76.92 \%$ son hombres (10).

Del total de académicos el $76.92 \%$ cuenta con un Doctorado (10) y el $23.07 \%$ tiene Maestría (3). Cada uno de los profesores colabora y suma a una de las tres Líneas de Generación y Aplicación del Conocimiento del Posgrado.

c) Apoyo al estudiante

En esta línea, el Cechiver tiene como objetivo incrementar y fortalecer las estrategias de apoyo a los estudiantes que aseguren un acompañamiento efectivo en atención a sus necesidades, desde su ingreso, permanencia y egreso-titulación, coadyuvando a la terminación oportuna de sus estudios para su inserción al mercado laboral.

Entre las acciones que realiza la Maestría destaca la participación en la EXPO Orienta, los días 21 y 22 de octubre de 2019, en donde se dio a conocer la oferta educativa.

Otros espacios donde la maestría se dio a conocer son:

Evento organizado por la Secretaría de Desarrollo Económico y Portuario del Estado de Veracruz (SEDECOP) y la Secretaría de Desarrollo Agropecuario, Rural y Pesca (SEDARPA) el 27 de noviembre de 2019.

Evento on-line organizado por COPARMEX el 29 de mayo de 2020.

Orientando. Temas de Asia Oriental. Sociedad, Cultura y Economía. Revista editada por el Centro de Estudios China-Veracruz de la Universidad Veracruzana, México (Centro de Estudios APEC) / año 10 / número 20/ 
Entre otras actividades que destacaron en este rubro fue la realización de un Webinar semanal Cechiver Conmex-CEAPEC, con especial énfasis para la participación de nuestros alumnos, sobre temas relacionados con las diversas experiencias educativas dictados por integrantes del Centro o invitados especiales.

Se estimula la participación en el dictado de conferencias y foros que desarrolla la Red ALC-China, de la que nuestro Centro es miembro, y que también tienen una frecuencia semanal.

En cuanto a la atención al desempeño de los estudiantes, se asignó a cada uno de los alumnos un académico que fungirá como su tutor a lo largo de su estancia. También, les fue designado un Director de Tesis y un Asesor, esto con la finalidad de que cuenten con asesoría especializada en la temática de su elección y con ello logren culminar sus estudios de manera satisfactoria, titulándose en el plazo establecido en los reglamentos institucionales.

\section{d) Investigación, innovación y desarrollo tecnológico}

Cechiver se propuso impulsar la innovación a través de formas de organización del quehacer institucional que optimicen la respuesta de la Universidad ante su compromiso social de generar conocimientos y tecnologías socialmente pertinentes, que favorezcan el desarrollo científico, tecnológico, económico, cultural y político de su entorno regional, estatal y nacional (Crónica de Xalapa, 09 de diciembre de 2019).

En las actividades realizadas por el cuerpo académico de MESCAL, se obtuvieron los siguientes resultados entre septiembre de 2019 y agosto de 2020:

- Se brindó asistencia constante a través de tutorías a 26 estudiantes.

- Dirección de seis tesis.

- Publicación de tres libros, dos capítulos de libros, dos artículos y dos libros en prensa.

Orientando. Temas de Asia Oriental. Sociedad, Cultura y Economía. Revista editada por el Centro de Estudios China-Veracruz de la Universidad Veracruzana, México (Centro de Estudios APEC) / año 10 / número 20/ 
- También para divulgar las investigaciones realizadas, Cechiver participó de seminarios internacionales, foros, dictado de conferencias en el país y en el exterior, webinarios y presentación de libros.

\section{Eje 2: Visibilidad e impacto social}

A través de este eje se busca proyectar a la Universidad Veracruzana como una institución con reconocimiento regional, nacional e internacional por su compromiso con el desarrollo social comunitario, humanístico, ambiental y cultural. Además incrementar y fortalecer la vinculación universitaria, la equidad de género, interculturalidad, sustentabilidad, internacionalización, inclusión, promoción de la salud, derechos humanos, justicia y artecreatividad; la participación en proyectos con el sector externo y su contribución a la formulación de políticas públicas. Asimismo, la promoción de la cultura de la paz y de los derechos humanos como parte de la responsabilidad social de la Institución.

Nuestro Centro desarrolló una intensa actividad en la región y en China con instituciones públicas y privadas tendientes a mejorar la calidad de vida de la sociedad civil veracruzana, especialmente a partir del fortalecimiento de las pequeñas y medianas empresas.

Algunos eventos han tenido influencias positivas en el corto plazo como es el caso de las tareas de capacitación para mejorar las condiciones de prestadores de servicios turísticos (Reyes Díaz, 01 de diciembre de 2019).

Se establecieron mecanismos de cooperación participando en diversos eventos, con las Secretarías de Desarrollo Económico y Portuario y la de Turismo del Estado, la Legislatura de Veracruz, el Senado de la República, la Secretaría de Economía, CANACINTRA, COPARMEX, ABAC y la MEXCHAM, Cámara de Comercio de México en China y cuya Presidencia del Capítulo Veracruz es ejercida por el Coordinador de Cechiver.

Nuestro organismo asesora a empresas de Veracruz y otros estados mexicanos para implementar el desarrollo de la economía digital propiciando la utilización del e-commerce.

Orientando. Temas de Asia Oriental. Sociedad, Cultura y Economía. Revista editada por el Centro de Estudios China-Veracruz de la Universidad Veracruzana, México (Centro de Estudios APEC) / año 10 / número 20/ 
En el año calendario que se evalúa, se fortaleció la presencia internacional de nuestra institución. En este sentido se destaca el Tercer Seminario sobre China y América Latina y el Caribe: Diálogo entre Civilizaciones, realizado en la ciudad de Xuxzou, China, en el que nuestro Centro fue coorganizador, y la Doctora Sara Ladrón de Guevara González dictó la conferencia: Territorios Lejanos, Tradiciones Vecinas; además de las presentaciones de los doctores Esteban Zottele y Aníbal Carlos Zottele.

Asimismo, el Centro de Estudios China-Veracruz realizó el XII Diplomado presencial sobre Cultura y Negocios en China en las ciudades de Shanghái y Changzhou. En su primera fase se valió de la plataforma EMINUS para generar las condiciones propicias que ayudaran a los participantes a definir con mayor precisión los términos del intercambio con organismos académicos, empresariales y del sector público de aquel país. Esta actividad, se desarrolló del 09 al 23 de octubre de 2019, y fue previa a la Segunda Exposición Internacional de Importaciones de China (CIIE), feria celebrada anualmente cada noviembre en la ciudad de Shanghái (Villa Ortiz, 16 de octubre de 2019; 18 de octubre de 2019 y 25 de octubre de 2019).

Como parte del primer ciclo de conferencias del Centro de Estudios China-Veracruz, cada viernes, desde el 26 de junio del presente año hasta el 18 de septiembre han tenido y tendrán lugar los siguientes los siguientes webinarios (Hermida Rosales, 01 de julio de 2020; 09 de julio de 2020; 20 de julio de 2020; 07 de agosto de 2020; Cortés Pérez, 15 de julio de 2020):

26 de junio de 2020

Veracruz y la Nao de China.

Lic. Guo Xiao Na, Licenciada en Letras Hispánicas, Universidad de Estudios Internacionales de Beijing.

03 de julio de 2020

Orientando. Temas de Asia Oriental. Sociedad, Cultura y Economía. Revista editada por el Centro de Estudios China-Veracruz de la Universidad Veracruzana, México (Centro de Estudios APEC) / año 10 / número 20/ 
El comercio más sano en tiempos de pandemia: e-commerce.

Lic. Claudia Elilú Méndez Viveros, Licenciada en Gestión y Dirección de Negocios.

Colaboradora del Centro de Estudios China-Veracruz. Universidad Veracruzana.

10 de julio de 2020

Entre Veracruz y Pekín: armamento naval en el inicio de la modernidad, un tema de la Global History.

Lic. Eder Antonio de Jesús Gallegos Ruiz, Historiador naval.

17 julio de 2020

Reformas en el ambiente de negocios en China y su impacto en reportes del Banco Mundial.

Mtra. Silvia Carolina López Rocha, Abogada especialista en Clima de Inversión. Banco Mundial.

24 de julio de 2020

China, un mercado de oportunidades para las Pymes mexicanas.

Mtro. José Luis Valencia Montano, Director General del Consejo Empresarial

Mexicano de Comercio Exterior, Inversión y Tecnología (COMCE), Capítulo Estado de Veracruz.

Mtro. Mario Alberto Santiago Mendoza, Secretario Técnico del Centro

de Estudios China-Veracruz, Centro de Estudios de APEC de la

Universidad Veracruzana.

Orientando. Temas de Asia Oriental. Sociedad, Cultura y Economía. Revista editada por el Centro de Estudios China-Veracruz de la Universidad Veracruzana, México (Centro de Estudios APEC) / año 10 / número 20/ 
Mtro. Víctor Manuel Herrera Mendoza, Assistant manager - CW

CPA, Latin Department./Subgerente - CW CPA, Departamento Latino.

21 de agosto de 2020

Características del turismo emisor chino que lo convierte en un mercado potencial para México.

Lic. Rubí del Ángel Herrera. Consultora autorizada por la Secretaría de Turismo Federal en el Sello Cerca de China.

28 de agosto de 2020. Inicio del ciclo Aproximaciones a la Franja y la Ruta. (cuatro semanas)

Teorías de las Relaciones Internacionales chinas.

Dr. Andrés Bórquez

Coordinador del Programa de Estudios Chinos de la Universidad de Chile, Doctor en Políticas Internacionales de la Universidad de Fudan, China.

4 de septiembre 2020

Ejes de integración y desarrollo Sudamericanos e Iniciativa la Franja y la Ruta: convergencia y proyección

Dr. Miltón Reyes

Coordinador del Centro de Estudios Chinos del Instituto de Altos Estudios Nacionales del Ecuador.

Orientando. Temas de Asia Oriental. Sociedad, Cultura y Economía. Revista editada por el Centro de Estudios China-Veracruz de la Universidad Veracruzana, México (Centro de Estudios APEC) / año 10 / número 20/ abril-septiembre 2020 / ISSN: 2007-5723 
11 de septiembre 2020

La Iniciativa de la Franja y la Ruta: entre el espejo del pasado y la imagen del futuro

Dr. Ignacio Villagrán

Director del Centro de Estudios Argentina-China (CEACh) de la Facultad de Ciencias Sociales (FSOC) de la Universidad de Buenos Aires (UBA)

18 de septiembre 2020

Presentación del Libro Aproximaciones a la Franja y la Ruta

Autores:

Dr. Esteban Zottele, Investigador y Docente de la Universidad de Changzhou, Representante de la Universidad Veracruzana en China.

Dr. Aníbal Carlos Zottele, Coordinador de Cechiver, Secretario Técnico de ConmexCEAPEC.

Entre los asistentes a nuestras sesiones virtuales se destaca una amplia participación estadual y nacional que revela un interés creciente hacia los temas sobre China y la región Asia-Pacífico, favoreciendo a la vez, a la divulgación de las investigaciones y publicaciones que realizamos en torno a la economía, cultura y sociedad de aquel país y de las 21 economías que integran APEC así como sus vínculos con distintas regiones de México y con otros países de América Latina y el Caribe.

\section{Vinculación y responsabilidad social universitaria}

Orientando. Temas de Asia Oriental. Sociedad, Cultura y Economía. Revista editada por el Centro de Estudios China-Veracruz de la Universidad Veracruzana, México (Centro de Estudios APEC) / año 10 / número 20/ 
Este ítem tiene como objetivo impulsar una permanente relación con los sectores público y empresarial que contribuyan al desarrollo de las funciones institucionales; fortalecer y extender la cultura universitaria en el ámbito estatal, nacional e internacional, a través de las actividades artísticas, científicas y académicas y la generación de contenidos.

Una de las importantes funciones para la vinculación entre organismos e instituciones es el nombramiento de la UV como Sede del Consorcio Mexicano de Centros de Estudios de APEC (Conmex-CEAPEC), y con ello, el 27 de marzo del 2020, la Presidencia y la Secretaría Técnica para el período 2020-2021. El CONMEX-CEAPEC es una asociación de Centros de Estudios de APEC a la que pertenecen diversas universidades del país, dentro de sus objetivos se encuentran promover la cooperación de la educación superior y la investigación avanzada, y propiciar la colaboración con los sectores gubernamental, social y empresarial.

Esta actividad se ejerce a plenitud a partir del 27 de marzo de 2020. Como parte de la gestión, se han fortalecido los vínculos con la Secretaría de Economía, con las universidades miembros del Consorcio (Universidad Autónoma de México, Universidad de Guadalajara, Universidad de Colima, Universidad Autónoma de Baja California Sur, Universidad Michoacana de San Nicolás de Hidalgo, Instituto Tecnológico y de Estudios Superiores Campus Guadalajara), y con las universidades que están en proceso de unirse al mismo (Universidad Autónoma de Nayarit, Universidad Autónoma de Nuevo León, Universidad Autónoma Metropolitana- Azcapotzalco, Universidad del Mar). Se propuso y se aprobó el Reglamento Interno del Consorcio, con la finalidad de establecer lineamientos durante la gestión que nuestra universidad tiene a cargo. Y, para fortalecer los vínculos con el sector empresarial, instituciones, organismos no lucrativos y gobierno a nivel nacional e internacional, se crearon dos Comisiones dentro del Consorcio: la Comisión de Relaciones Internacionales y la Comisión de Relaciones Socioeconómicas. De esta manera, la Universidad Veracruzana tiene un papel definido en la generación de investigaciones, propuestas y discusión de las distintas instancias que desarrolla este Foro (Sandoval Rodríguez, 25 de marzo de 2020, 27 de marzo de 2020; Peralta Vázquez, 16 de mayo de 2020; Herrera Gómez, 09 de abril de 2020).

Orientando. Temas de Asia Oriental. Sociedad, Cultura y Economía. Revista editada por el Centro de Estudios China-Veracruz de la Universidad Veracruzana, México (Centro de Estudios APEC) / año 10 / número 20/ 


\section{Emprendimiento y egresados}

El objetivo para esta línea de acción del plan estratégico institucional es fortalecer y extender el espíritu emprendedor y la cultura empresarial a través de la promoción de actividades de formación integral universitaria, respondiendo a las necesidades del entorno y contribuyendo al desarrollo económico y social; conocer la actuación de los egresados en el mundo laboral, su percepción por la formación recibida y la opinión de los empleadores sobre su desempeño para enriquecer los planes y programas de estudio

Destaca la titulación, aplicando la modalidad virtual, de dos de los egresados de la MESCAL. También, se elaboró y aplicó encuesta a los egresados del Posgrado con la finalidad de conocer sus opiniones acerca del impacto de su formación en el ámbito laboral, y los motivos por los cuales algunos no han concluido con su proceso de titulación, esto en el marco del Programa Institucional de Seguimiento de Egresados (PISE) durante julio de 2020.

\section{Cultura humanista y desarrollo sustentable}

Como parte de las actividades desarrolladas para favorecer la cultura humanista, se efectuó la presentación del libro "Veracruz: de la Nao de China a la Franja y la Ruta", hecho en el marco de la conmemoración del 500 aniversario de la fundación de Veracruz, en el que se incluyeron artículos de la autoría de estudiantes de la MESCAL.

De igual manera, se han organizado y coordinado distintas presentaciones y conferencias a través de los Webinarios que genera el Cechiver, en los que se desarrollaron distintos temas sobre áreas de economía, cultura y sociedad de China y su vínculo con México.

\section{Internacionalización e interculturalidad}

Durante el período de gestión la Universidad Veracruzana con la participación de nuestro Centro estableció y renovó convenios con instituciones académicas de China, destacan: la Academia de Ciencias de China, la Academia de Ciencias Sociales de Shanghái, Renmin University, Beijing International Studies University, los Institutos de Relaciones

Orientando. Temas de Asia Oriental. Sociedad, Cultura y Economía. Revista editada por el Centro de Estudios China-Veracruz de la Universidad Veracruzana, México (Centro de Estudios APEC) / año 10 / número 20/ 
Internacionales Contemporáneas de China (CICIR), la Universidad Normal de Xuzhou y la Universidad de Changzhou.

Los convenios con esas instituciones académicas contribuyen al fortalecimiento de actividades culturales, científicas y tecnológicas.

Además, se encuentra en su fase final el acuerdo se establecieron con Hanban (Institutos Confucio) que permitirá en un futuro próximo, la presencia de ese organismo en la ciudad de Veracruz.

En lo inmediato, los vínculos con la Universidad de Changzhou, han facilitado el dictado en condiciones favorables de nuestro diplomado Cultura y Negocios en China que se realiza anualmente en aquel país, así como el fomento de la internacionalización e interculturalidad a través de la conferencia dictada por la Decana de la Facultad de Lengua Hispánica de esta Universidad, Dra. Wei Quian, el 6 de diciembre de 2019, en el Puerto de Veracruz; convocando a estudiantes de la UV y a empresarios.

Con la cooperación del representante de la UV en China y de nuestro centro las autoridades de la Editorial de esta casa de estudios y de la Feria Internacional del Libro Universitario, visitaron China y profundizaron sus vínculos con diversos organismos de ese país con avances significativos en relación con la actividad de publicaciones, relacionadas con la difusión recíproca de investigaciones y de conocimiento sobre ambas culturas.

\section{Eje 3: Gestión y gobierno}

Según lo establecido por el Programa de Trabajo Estratégico 2017-2021, este eje tiene como propósito garantizar la operación de las funciones sustantivas y adjetivas, disponiendo de los recursos humanos y financieros necesarios además de un gobierno moderno desconcentrado. En cumplimiento con estas disposiciones, la administración del Cechiver incorporó cada una de las innovaciones establecidas por la Secretaría de Administración y Finanzas.

\section{Financiamiento}

Orientando. Temas de Asia Oriental. Sociedad, Cultura y Economía. Revista editada por el Centro de Estudios China-Veracruz de la Universidad Veracruzana, México (Centro de Estudios APEC) / año 10 / número 20/ 
Se optimizaron los recursos asignados y se gestionaron otros que representaron un monto aproximado similar a la asignación del presupuesto ordinario, que fueron aplicados a la internacionalización (evento desarrollado en China) y publicaciones (libro De la Nao de China a la Franja y la Ruta) ambos financiados íntegramente por usuarios de nuestros servicios y por empresas.

\section{Infraestructura física y tecnológica}

Como parte de la ampliación de la infraestructura física que compone al Centro de Estudios China-Veracruz, se inauguró una oficina en la sede de la Facultad de Contaduría de la región Veracruz, lo que ha permitido realizar labores presenciales de investigación y académicas, que ayudan a fortalecer la vinculación empresarial. Es, también, una alternativa más para los jóvenes estudiantes de la Universidad Veracruzana y de otras instituciones de educación superior en el desarrollo de sus prácticas profesionales y de servicio social (Martínez y Enríquez, 25 de abril de 2019).

Conjuntamente, se gestionó la adquisición de equipo tecnológico para el nuevo personal adscrito al Cechiver y durante los meses de abril y mayo de 2020, se trabajó en la actualización de los contenidos de los diferentes módulos que integran el diplomado Negocios entre China y México en la plataforma EMINUS.

Se complementaron los servicios tecnológicos que este Centro de Estudios administra, en el transcurso del 2020, con la creación de nuevas redes sociales y la reactivación de las existentes, las cuales sirven como mecanismos que permiten informar sobre las distintas actividades que el Centro realiza y favorecer la difusión de la oferta educativa y webinarios que dicta el Cechiver. También se realizó un plan de ampliación de nuestra presencia en las redes sociales, como Facebook, Instagram, Twitter, y la página web con la finalidad de generar un mayor alcance y una interacción más cercana con los seguidores.

- Facebook: CECHIVERUV

- Instagram: CECHIVER_UV

- Twitter: CECHIVER UV

Orientando. Temas de Asia Oriental. Sociedad, Cultura y Economía. Revista editada por el Centro de Estudios China-Veracruz de la Universidad Veracruzana, México (Centro de Estudios APEC) / año 10 / número 20/ 
- Página web: https://www.uv.mx/chinaveracruz

En cuanto a los medios tecnológicos que se utilizan para realizar las actividades concernientes a la secretaría técnica del Consorcio Mexicano de Centros de Estudios APEC (Conmex-CEAPEC) el Cechiver diseñó un plan de trabajo a través del uso de medios de comunicación para tener presencia en redes sociales: Se compró el dominio de una página web, la cual fue programada y diseñada con información actualizada, integrándosele, a su vez, la información que tenía el anterior sitio web del Consorcio. El objetivo de estas redes sociales y de la página web del Conmex-CEAPEC es posicionar dichas plataformas para favorecer la interacción con los seguidores y permita mantenerlos informados sobre las actividades que se están realizando.

Las redes sociales que administra el Cechiver como Centro de Estudios de APEC y ejerciendo las funciones técnicas del Conmex-CEAPEC son las siguientes:

Facebook: conmexceapec

Instagram: conmexceapec

Twitter: conmexceapec

Página web: https://www.conmexceapec.org/

Correos: contacto@conmexceapec.org/conmexceapec@gmail.com

Además, se crearon los correos electrónicos oficiales con los que se intercambia información. Todo esto con el fin de tener una mejor comunicación con dichos miembros y poder informar las actividades que cada Centro de Estudios realiza, así como difundir la oferta educativa, publicaciones y noticias que ofrece cada institución.

Orientando. Temas de Asia Oriental. Sociedad, Cultura y Economía. Revista editada por el Centro de Estudios China-Veracruz de la Universidad Veracruzana, México (Centro de Estudios APEC) / año 10 / número 20/ 


\section{Conclusiones}

El periodo septiembre 2019-agosto 2020 estuvo condicionado por los acontecimientos conocidos, pero el Cechiver pudo cumplir con una parte sustantiva de las actividades trazadas, porque entre sus antecedentes se encuentra el haber utilizado la plataforma Eminus para el dictado de su Maestría y de los diplomados, a excepción del que se desarrolla desde doce años atrás en China.

Se multiplicaron los eventos virtuales y semanalmente, junto a las actividades de dictados de las experiencias educativas y a las tres reuniones que sostiene todo el equipo de trabajo, se agregaron los encuentros con instituciones académicas, organismos empresariales y del sector público con los que se interactúa, tanto en espectos relacionados con las investigaciones que desarrolla el Centro como con los acuerdos referidos a las asesorías en comercio e inversión entre México y China.

El trabajo responsable y sistemático se expresa en resultados. A finales del 2019, nuestra Universidad fue designada como sede del Consorcio de Centros de Estudios de APEC, luego de varios años de pertenecer a esa estructura, que tiene como exigencia el aval de las Secretarías de Economía, de la Cancillería y de las autoridades de APEC con sede en Singapur. De esta forma hasta fines del próximo año nuestra Universidad es el vínculo directo con todas las Universidades que integran los consorcios de las 21 economías que participan de esta organización.

Las actividades con instituciones académicas chinas y nuestras investigaciones nos han permitido una relación privilegiada con algunas de ellas. En tal sentido se destaca la próxima publicación de un libro coordinado por el Director del Think Tank de la Academia de Ciencias Sociales del Shanghái (SASS) y el Coordinador de Cechiver (documento con firma del acuerdo anexo), que será publicado a la brevedad por la Editorial Palgrave y financiado en su totalidad por SASS.

La Covid-19 ha generado una nueva situación a nivel económico y social que demanda un mayor esfuerzo de las instituciones académicas. La sociedad civil reclama, de organismos Orientando. Temas de Asia Oriental. Sociedad, Cultura y Economía. Revista editada por el Centro de Estudios 
como las universidades, respuestas a los problemas de desempleo, de pobreza extrema, de desaparición de pequeñas y medianas empresas, y de aumentos a los desequilibrios entre regiones.

Por eso, en el marco del Programa de Trabajo Estratégico 2017-2020, en el próximo período trabajaremos en forma coordinada con los sectores públicos y privados que reconozcan estas exigencias.

\section{Referencias}

Cortés Pérez, P. (15 de julio de 2020). Historias locales explican la globalización: Eder Gallegos. Universo. https://www.uv.mx/prensa/banner/historias-locales-explican-la-globalizacioneder-gallegos/

Crónica de Xalapa. (09 de diciembre de 2019). Presentan el libro "Veracruz: De la Nao de China a la Franja y la Ruta". Crónica de Xalapa. http://cronicadexalapa.com/presentan-el-libroveracruz-de-la-nao-de-china-a-la-franja-y-la-ruta/

Herrera Gómez, I. (09 de abril de 2020). Se celebra la primera reunión anual del Consorcio Mexicano de Centros de Estudios de APEC. Conmex-CEAPEC. https://www.conmexceapec.org/noticias/se-celebra-la-primera-reunion-anual-del-consorciomexicano-de-centros-de-estudios-apec/

Hermida Rosales, C. H. (01 de julio de 2020). Veracruz puede volver a ser puerta del comercio internacional: Guo Xiao Na. Universo. https://www.uv.mx/prensa/general/veracruz-puedevolver-a-ser-puerta-del-comercio-internacional-guo-xiao-na/

- (09 de julio de 2020). El e-commerce gana terreno en México: Claudia Méndez. Universo. https://www.uv.mx/prensa/banner/el-e-commerce-gana-terreno-en-mexico-claudia-mendez/

- (20 de julio de 2020). China y el Banco Mundial cumplen 40 años de cooperación. Universo. https://www.uv.mx/prensa/banner/china-y-el-banco-mundial-cumplen-40-anos-decooperacion/

-. (07 de agosto de 2020). China posee mercado importante para productos novedosos: expertos. Universo. $\quad$ https://www.uv.mx/prensa/banner/china-posee-mercado-importante-paraproductos-novedosos-expertos/

Martínez, A y Enríquez, S. (25 de abril de 2019). Cechiver inauguró oficina en la región Veracruz. Universo. https://www.uv.mx/prensa/regiones/veracruz/cechiver-inauguro-oficina-en-laregion-veracruzl

Peralta Vázquez, C. (16 de mayo de 2020). UV presidió reunión extraordinaria del ConmexCEAPEC. Conmex-CEAPEC. https://www.conmexceapec.org/noticias/uv-presidio-reunionextraordinaria-del-conmex-ceapec/

Reyes Díaz, K. (01 de diciembre de 2019). Cechiver-UV capacitó a prestadores de servicios turísticos en Veracruz. Universo. https://www.uv.mx/prensa/banner/cechiver-uv-capacito-aprestadores-de-servicios-turisticos-en-veracruz/

Sandoval Rodríguez, D. (25 de marzo de 2020). UV será la sede del Consorcio Mexicano de Centros de Estudios de APEC. Conmex-CEAPEC. https://www.conmexceapec.org/noticias/uv-serala-sede-del-consorcio-mexicano-de-centros-de-estudios-apec/

Orientando. Temas de Asia Oriental. Sociedad, Cultura y Economía. Revista editada por el Centro de Estudios 
- (27 de marzo de 2020). UV asumió presidencia y secretaría técnica del Consorcio Mexicano de Centros de Estudio del Foro de Cooperación Económica Asia Pacífico (APEC). ConmexCEAPEC. https://www.conmexceapec.org/noticias/uv-asumio-presidencia-de-asambleaanual-del-conmex-ceapec/

Villa Ortiz, I (16 de octubre de 2019). UV enriquece las relaciones entre México y China: Lorena Larios Rodríguez. Universo. https://www.uv.mx/prensa/banner/uv-enriquece-las-relacionesentre-mexico-y-china-lorena-larios-rodriguez/

- $(18$ de octubre de 2019). Veracruz cautiva a Changzhou. Universo. https://www.uv.mx/prensa/banner/veracruz-cautiva-a-changzhou/

- (25 de octubre de 2019). Cechiver-UV se reafirma como vía para llegar a China. Universo. https://www.uv.mx/prensa/banner/cechiver-uv-se-reafirma-como-via-para-llegar-a-china/

Orientando. Temas de Asia Oriental. Sociedad, Cultura y Economía. Revista editada por el Centro de Estudios China-Veracruz de la Universidad Veracruzana, México (Centro de Estudios APEC) / año 10 / número 20/ 